\title{
Fornix Transection Impairs Learning of Randomly Changing Object Discriminations
}

\author{
Charles R. E. Wilson, David P. Charles, ${ }^{\dagger}$ Mark J. Buckley, and David Gaffan \\ Department of Experimental Psychology, Oxford University, Oxford OX1 3UD, United Kingdom
}

\begin{abstract}
The hippocampus has a well established role in spatial memory, but increasing evidence points to a role in nonspatial aspects of memory. To investigate such a role, six macaque monkeys received a bilateral transection of the fornix to disconnect subcortical inputs and outputs of the hippocampus. An additional six macaque monkeys constituted an unoperated control group. To test the involvement of the hippocampus in nonspatial aspects of memory, both groups were trained postoperatively on four concurrent visual object discrimination problems, each problem having one rewarded object and one unrewarded. After acquisition to criterion of these discriminations, the monkeys learned five subsequent stages of discriminations using the same objects. In each of these stages, both the pairings of objects one with another, and the reward assignments for the objects, were randomly reassigned. In the initial acquisition stage, control and fornix animals were equally proficient in learning the discriminations. In the five reassigned stages, however, monkeys with fornix transection made on average three times as many errors as the controls in learning the discriminations. This impairment was noted even in trials where the reward assignments from the previous stage were maintained in the new stage. These findings are consistent with other recent evidence for a role beyond the spatial domain for the fornix in monkeys.
\end{abstract}

Key words: fornix; monkey; hippocampus; memory; macaque; spatial

\section{Introduction}

The hippocampus has a well established role in spatial memory. Impairments in spatial memory in monkeys with transection of the fornix, a major input/output pathway of the hippocampus, have strongly contributed to this view (Murray et al., 1989; Gaffan, 1994a; Buckley et al., 2004). More recently, the fornix in the monkey has also been implicated in aspects of nonspatial memory (Brasted et al., 2002, 2003), and such work supports a number of views of hippocampal function which describe a more general role than that limited to spatial processing (Rudy and Sutherland, 1995; Eichenbaum and Cohen, 2001).

Several demonstrations of nonspatial impairments after hippocampal or fornix damage have implicated these structures in memory for temporal context. Rats with hippocampal lesions show impairments in recalling the relative positions of items presented in a sequence (Fortin et al., 2002), and in disambiguating more than one sequence (Agster et al., 2002), and monkeys with transection of the fornix are impaired at making judgements of relative recency, but not of absolute novelty (Charles et al., 2004).

These studies suggest that even nonspatial memory tasks may be impaired after selective damage to the fornix or hippocampus in monkeys, provided they place some demand on processing of

Received Aug. 3, 2007; revised 0ct. 9, 2007; accepted 0ct. 10, 2007.

This work was supported by the United Kingdom Medical Research Council. C.R.E.W. was supported by the Christopher Welch Trust. We would like to express our sadness at the death of our much-missed colleague, David P. Charles.

${ }^{\dagger}$ Deceased, 0ct. 17, 2006

Correspondence should be addressed to Charles R. E. Wilson, Department of Experimental Psychology, Oxford University, South Parks Road, 0xford OX1 3UD, UK. E-mail: charles.wilson@psy.ox.ac.uk.

DOI:10.1523/JNEUROSCI.3536-07.2007

Copyright $\odot 2007$ Society for Neuroscience $\quad 0270-6474 / 07 / 2712868-06 \$ 15.00 / 0$ temporal context. Simple forms of associative learning such as concurrent object discrimination learning may be unimpaired by selective hippocampal (Teng et al., 2000) or fornix (Moss et al., 1981; Gaffan, 1994b) lesions in monkeys, but impairments in concurrent object discrimination learning may emerge when learning of the discrimination problems places demands on temporal context.

We tested this hypothesis in macaque monkeys with fornix transection using a series of concurrent object discriminations. After an initial acquisition phase of four pairs of object discriminations, the monkeys learned five subsequent stages of discriminations using the same objects. In each of these subsequent stages, both the pairings of objects one with another, and the reward assignments for the objects, were randomly reassigned. Previous experiments on the reassignment of reward contingencies or the repairing of stimuli present conflicting results. Rats with fornix transection are impaired when pairs are reassigned in paired associate learning (Eichenbaum et al., 1989), but not under all conditions (Deacon and Rawlins, 1996), and are facilitated in reversal learning (Eichenbaum et al., 1986).

An important feature of the current design was that the reward contingencies of individual objects did not necessarily change between stages, but the context in which the objects were presented, such as the identity of the other object, generally did. The random reassignment of stimuli between stages may result in problems in which the response-reinforcement contingencies of individual items have not changed, and any impairment in such problems could not be interpreted in terms of theories based around the inflexibility of reward value assignments. Thus, although the task does not include an explicit requirement to remember temporal context, one of the differences between this 


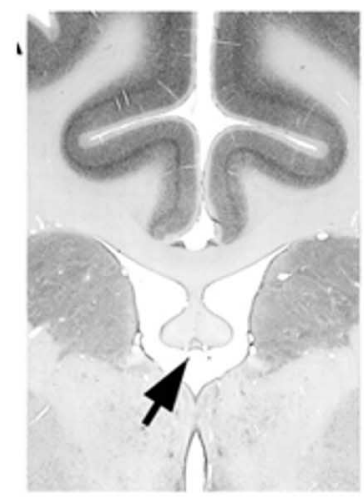

Fx 1

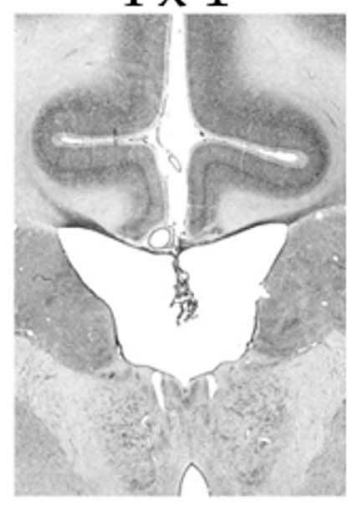

Fx 4

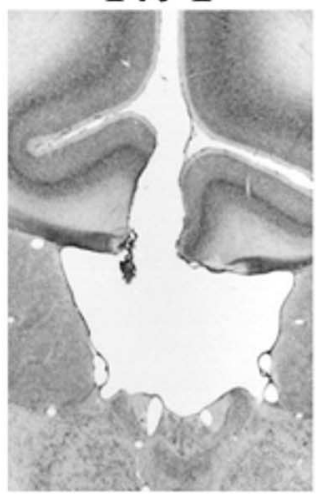

Fx 2

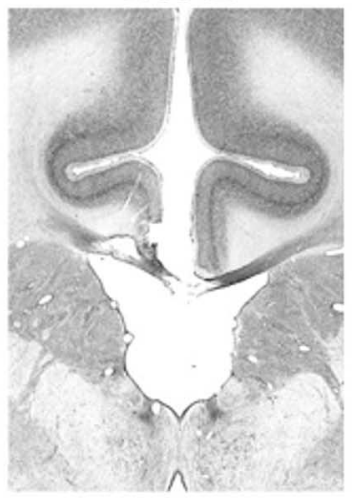

Fx 5

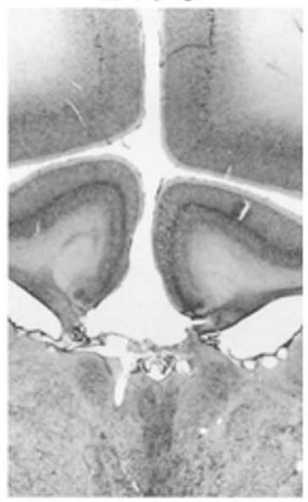

Fx 3

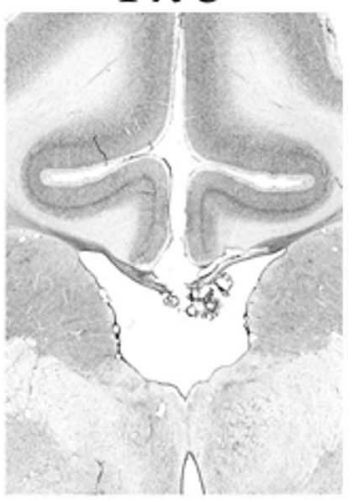

Fx 6

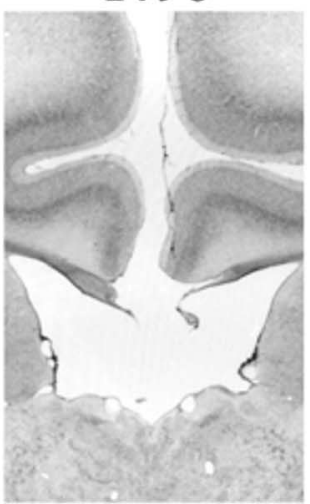

Figure 1. Coronal sections from the brains of a normal monkey (top), where the arrow indicates the intact fornix, and monkeys Fx 1-6, where the fornix has been completely transacted at the level of the interventricular foramen.

task and a series of concurrent object discriminations is an alteration in temporal context between stages.

\section{Materials and Methods}

Subjects. Twelve male macaque monkeys formed two experimental groups. One group (Fx 1-6) had received bilateral fornix (Fx) transection, and the remaining six monkeys (Con 1-6) acted as unoperated controls (Con). Each group contained three cynomolgus macaques ( $\mathrm{Ma}$ caca fascicularis) and three rhesus macaques (Macaca mulatta). All six rhesus monkeys had the same experimental history in the touch screen apparatus (see below), involving a series of visual discrimination learning tasks (Buckley et al., 2004; Charles et al., 2004). Similarly, all six cynomolgus monkeys had the same previous experimental history, also in the present apparatus, and also in a visual discrimination learning task. At no point in the current experiment was there a significant difference in performance between the two species (see below, Statistical analysis).

The monkeys' mean weight was $4.9 \mathrm{~kg}$ and their mean age was 5 years 1 month. All monkeys were housed socially in accordance with United Kingdom Home Office guidelines. Water was continually available in the home cage.

Surgery. Six monkeys received bilateral fornix transection (group Fx) and the remaining monkeys acted as unoperated controls (group Con). All procedures were performed under license in compliance with the United Kingdom Animals (Scientific Procedures) Act 1986. Operations were performed under sterile conditions. The monkeys were anesthetized throughout surgery with barbiturate (5\% thiopentone sodium, to effect) administered through an intravenous cannula. Monkeys were maintained in a state of deep anesthesia by monitoring of pulse rate, blood oxygenation, body temperature and peripheral reflexes. The transections were made by aspiration with the aid of an operating microscope. A D-shaped bone flap was raised over the midline and the left hemisphere. The dura mater was cut to expose the hemisphere up to the midline. Veins draining into the sagittal sinus were cauterized and cut. The left hemisphere was retracted from the falx with a brain spoon. A glass aspirator was used to make a sagittal incision no $>5 \mathrm{~mm}$ in length in the corpus callosum at the level of the interventricular foramen. The fornix was sectioned transversely by electrocautery and aspiration with a 20 gauge metal aspirator, which was insulated to the tip. The dura mater was drawn back but not sewn, and the bone flap replaced. When the lesion was complete, overlying tissue was closed in layers. Monkeys received buprenorphine hydrochloride $(0.01 \mathrm{mg} / \mathrm{kg})$ as an analgesic and amoxicillin $(8.75 \mathrm{mg} / \mathrm{kg})$ to prevent infection, daily, for 1 week postoperatively under veterinary guidance.

Histology. After the conclusion of the current experiment, the monkeys with fornix transection were deeply anesthetized with sodium pentobarbitone and perfused through the heart with saline followed by $10 \%$ formalin solution. The brains were blocked in the coronal stereotaxic plane posterior to the lunate sulcus, removed form the skull, and allowed to sink in sucrose-formalin solution. The brains were cut in $50 \mu \mathrm{m}$ sections on a freezing microtome. Every fifth section was retained and stained with cresyl violet. Microscopic examination of the stained sections revealed in every case a complete section of the fornix (see Fig. 1) with no damage outside the fornix, except for the incision in the corpus callosum of each animal as described in the surgical procedures, and slight damage to the most ventral part of cingulate gyrus in one hemisphere in two animals (Fx 2 and Fx 4).

Apparatus. The experiment was conducted in an automated testing apparatus contained within an experimental cubicle that was dark except for the display background illumination. Monkeys were brought to the apparatus in a wheeled transport cage that was secured opposite a touch screen $380 \mathrm{~mm}$ wide and $280 \mathrm{~mm}$ high with a display resolution of $800 \times$ 600 pixels, on which the experimental stimuli (described below) were presented. Animals could reach through the horizontal bars at the front of the transport cage and touch the touch screen. An automated pellet dispensing device made an audible click when delivering banana flavored 
Table 1. Stimuli assigned, and their reward contingencies, in the acquisition stage and the five subsequent reassignment stages (R1-R5)

\begin{tabular}{|c|c|c|c|c|c|c|}
\hline Concurrent problem & Acquisition stage & Stage R1 & Stage R2 & Stage R3 & Stage R4 & Stage R5 \\
\hline 1 & $1+$ vs $6-$ & $\mathbf{P} 2+$ vs $3-$ & S $2+$ vs $8-$ & P $3+$ vs $6-$ & P $3+$ vs $8-$ & S $4+$ vs $8-$ \\
\hline 2 & $5+$ vs $7-$ & M $4+$ vs $8-$ & M 1+ vs 7- & S $1+$ vs $4-$ & M $6+$ vs $7-$ & P $6+$ vs $3-$ \\
\hline 3 & $2+$ vs $4-$ & S $5+$ vs $7-$ & v $3+$ vs $5-$ & V 5+ vs $2-$ & V $2+$ vs $1-$ & M 1+ vs 7- \\
\hline 4 & $3+$ vs $8-$ & V $6+$ vs $1-$ & P $6+$ vs $4-$ & M 8+vs $7-$ & v $4+$ vs $5-$ & S $2+$ vs $5-$ \\
\hline
\end{tabular}

The numbers $1-8$ represent a particular stimulus, and the number on the left is the rewarded stimulus $(+)$ whereas the number on the right is unrewarded $(-)$. Each column represents the stimulus pairings that constitute the block of concurrent trials that are presented in a given stage of testing. S, Same shift; $V$, reversal shift; $P$, plus shift; $M$, minus shift.

reward pellets (190 mg; P.J. Noyes, Lancaster, $\mathrm{NH}$ ) into a well positioned to the right at the foot of the screen. An automated spring loaded lunchbox (length $200 \mathrm{~mm}$, width $100 \mathrm{~mm}$, height $100 \mathrm{~mm}$ ) positioned to the left and at the foot of the touch screen opened immediately after the end of each daily session, determined by the completion of the required number of blocks (see below, Experimental procedure) and a final correct choice, to deliver the animals' daily diet of wet primate chow, pieces of fruit, dates, and peanuts. A closed-circuit TV camera positioned above the touch screen and in front of the monkey was used for observation from another room from which the stimulus display, food delivery, and experimental contingencies were computer-controlled.

Stimulus material. The visual stimuli presented on the touch screen were eight individual computer generated images. The images used were a white triangle, a white circle, a white cross, a black and white checker board, a blue square, a yellow square, a red square, and a green square. Each image occupied a maximum area of $8.5 \mathrm{~cm}$ high and $8.5 \mathrm{~cm}$ wide. All stimuli were presented against a black background that occupied the whole touch screen. A touch-sensitive area extending 50 pixels beyond each side of each image was used to ensure the detection of stimulus directed responses. Stimuli were randomly assigned to pairs and each member of each pair was randomly assigned as either rewarded or nonrewarded at the beginning of each stage of the experiment.

Experimental procedure. All monkeys were trained on a four-pair concurrent object discrimination task presented on the automated touch screen. At the beginning of the experiment and each of the subsequent five stages of testing, the eight objects were randomly assorted into four pairs, and in each object pair one object was randomly assigned as rewarded and the other as nonrewarded. For each stage of random repairing, the reward assignment changes were classified according to four possible different types of shift, determined by comparison with the reward assignments for each object during the previous stage. A same shift (Table 1) maintained the previous reward assignments, whereas a reversal shift reversed them. A plus shift comprised a new problem using two stimuli that were both rewarded in the previous stage, whereas a minus shift comprised a new problem using two stimuli that were both unrewarded in the previous stage. Table 1 below describes how these stimuli were arranged in the reassignment stages (Rs).

Within each stage of testing (Table 1, acquisition and R1-R5), the pairing of objects and the reward/nonreward assignment remained consistent across the daily sessions until animals had achieved a criterion of $90 \%$ correct in one session. The four object pairs formed a block of four trials and within each block the order of presentation of the object pairs was randomized. Each choice trial consisted of an object pair presented on the touch screen. Objects were presented in the vertical midline of the screen positioned equidistant either side of the central point and at least $100 \mathrm{~mm}$ apart. The left-right position of the two stimuli in a given problem was determined at random on each trial. Monkeys were required to touch one of the objects on the screen. Touching the rewarded object caused both stimuli to disappear from the screen as well as immediate production of a reward pellet. After an intertrial interval of $5 \mathrm{~s}$ the trial procedure was repeated with another object pair. Touching the nonrewarded object also caused both stimuli to disappear, but without a reward pellet, and caused the commencement of an intertrial interval of 10 s. Touching the screen during either intertrial interval reset that interval.

On day 1 of the first stage, all monkeys received 10 blocks of trials, and over a mean period of $4 \mathrm{~d}$ the number of trials in each daily session was increased until all monkeys received 50 blocks per day. The session terminated only when the monkey had made a final correct response, which triggered the automated lunchbox to open immediately and deliver the animal's daily diet. Training in each stage ended when a criterion of $90 \%$ correct responses was attained in a single session. The experiment lasted until all six stages had been completed by all animals.

Statistical analysis. The measure used was the number of errors taken by monkeys to reach the criterion of $90 \%$ correct in a single session. In the analysis described below, species of monkey was included as a factor. There were no significant effects of species, and no significant interactions with species. Hence it is clear that neither the slightly differing training history, nor the species of monkey had a significant effect on results.

\section{Results}

The overall results of the experiment are presented in Table 2, and graphically in Figure 2. In the initial acquisition stage, control and fornix animals did not differ significantly in the number of errors committed in the task $(t=1.067 ; \mathrm{df}=10 ; p=0.311)$. This indicates that fornix transection does not impair concurrent object discrimination learning before a demand on temporal context is introduced to the task, in agreement with previous findings (Moss et al., 1981; Gaffan, 1994b).

In the five reassigned stages, however, the monkeys with fornix lesions made significantly more errors relative to controls in learning the discriminations. ANOVA performed on the errors to criterion for R1-R5 showed a significant group effect $\left(F_{(1,9)}=\right.$ $11.2 ; p=0.010)$. There was no significant effect of the five different stages $(F<1)$ and no interaction between group and stage $(F<1)$. Importantly, comparison of the difference in errors between the acquisition stage and the mean of the five reassignment stages revealed a significant effect of lesion $\left(F_{(1,9)}=3.667\right.$; onetailed $p=0.046)$, supporting the prediction that monkeys with fornix transection would commit significantly more errors in the reassignment stages relative to the acquisition stage.

The data were also analyzed in terms of the type of shift performed in each trial. Table 3 shows mean errors for the different shift types, and Figure 3 presents these data graphically.

Although there were differences in the number of errors committed between the different types of shift, monkeys with fornix transection were significantly impaired on each type of shift, including "easy" shifts in which the reward assignments of the stimuli were maintained from the previous stage. ANOVA showed, as before, a significant main effect of lesion, as well as significant differences between shift types $\left(F_{(3,24)}=19.074 ; p<0.0005\right)$. The interaction between shift type and group did not quite reach statistical significance, although there was a clear trend toward such an interaction $\left(F_{(3,24)}=2.899 ; p=0.056\right)$. When considering individual shifts, it was clear in both groups that Reversal and Plus shifts elicited the most errors, whereas Minus shifts elicited the fewest errors of all. Nevertheless, comparison of each individual type of shift problem revealed a significant effect of fornix transection on each (Same, $F_{(1,9)}=10.17, p=0.013$; Reversal, $F_{(1,9)}=5.33, p=0.05$; Plus, $F_{(1,9)}=8.40, p=0.02$; Minus, $F_{(1,9)}$ $=6.96, p=0.03)$. Thus, although this trend toward an interaction likely reflects differences in the severity of the deficit across 
Table 2. Total errors and total trials to criterion for individual animals in the acquisition (A) and subsequent repairing (R1-R5) stages for animals with fornix transection (Fx 1-6) and controls (Con 1-6)

\begin{tabular}{|c|c|c|c|c|c|c|c|c|c|c|c|c|c|c|}
\hline & \multicolumn{2}{|l|}{ A } & \multicolumn{2}{|l|}{ R1 } & \multicolumn{2}{|l|}{ R2 } & \multicolumn{2}{|l|}{ R3 } & \multicolumn{2}{|l|}{ R4 } & \multicolumn{2}{|l|}{ R5 } & \multicolumn{2}{|c|}{$\mathrm{R} 1-\mathrm{R} 5$} \\
\hline & $\mathrm{E}$ & $\mathrm{T}$ & $\mathrm{E}$ & $\mathrm{T}$ & $E$ & $\mathrm{~T}$ & $E$ & $\mathrm{~T}$ & $E$ & $\mathrm{~T}$ & $E$ & $\mathrm{~T}$ & $\mathrm{E}$ & $\mathrm{T}$ \\
\hline Fx 1 & 13 & 120 & 42 & 480 & 14 & 200 & 139 & 600 & 7 & 200 & 8 & 200 & 210 & 1680 \\
\hline $\mathrm{F} \times 2$ & 38 & 240 & 35 & 360 & 101 & 544 & 39 & 400 & 10 & 200 & 118 & 600 & 303 & 2104 \\
\hline Fx 3 & 102 & 356 & 30 & 240 & 127 & 800 & 67 & 400 & 112 & 800 & 77 & 400 & 413 & 2640 \\
\hline Fx 4 & 19 & 200 & 55 & 400 & 19 & 200 & 73 & 600 & 90 & 800 & 79 & 600 & 316 & 2600 \\
\hline Fx 5 & 42 & 400 & 49 & 400 & 19 & 200 & 120 & 800 & 74 & 600 & 127 & 924 & 389 & 2924 \\
\hline Fx 6 & 18 & 200 & 33 & 400 & 38 & 400 & 33 & 400 & 37 & 400 & 14 & 200 & 155 & 1800 \\
\hline Mean & 39 & 253 & 41 & 380 & 53 & 391 & 79 & 533 & 55 & 500 & 71 & 487 & 298 & 2291 \\
\hline Con 1 & 16 & 121 & 3 & 120 & 11 & 160 & 3 & 200 & 5 & 200 & 15 & 200 & 37 & 880 \\
\hline Con 2 & 11 & 116 & 8 & 120 & 5 & 160 & 28 & 400 & 9 & 200 & 14 & 200 & 64 & 1080 \\
\hline Con 3 & 13 & 116 & 4 & 120 & 5 & 160 & 14 & 200 & 3 & 200 & 36 & 400 & 62 & 1080 \\
\hline Con 4 & 34 & 400 & 15 & 200 & 6 & 200 & 5 & 200 & 4 & 200 & 19 & 200 & 49 & 1000 \\
\hline Con 5 & 18 & 200 & 42 & 400 & 41 & 400 & 40 & 400 & 19 & 200 & 13 & 200 & 155 & 1600 \\
\hline Con 6 & 46 & 400 & 56 & 400 & 17 & 200 & 71 & 400 & 104 & 800 & 39 & 400 & 287 & 2200 \\
\hline Mean & 23 & 226 & 21 & 227 & 14 & 213 & 27 & 300 & 24 & 300 & 23 & 267 & 109 & 1307 \\
\hline
\end{tabular}

Measures include the criterial session. E, Total errors; T, total trials.

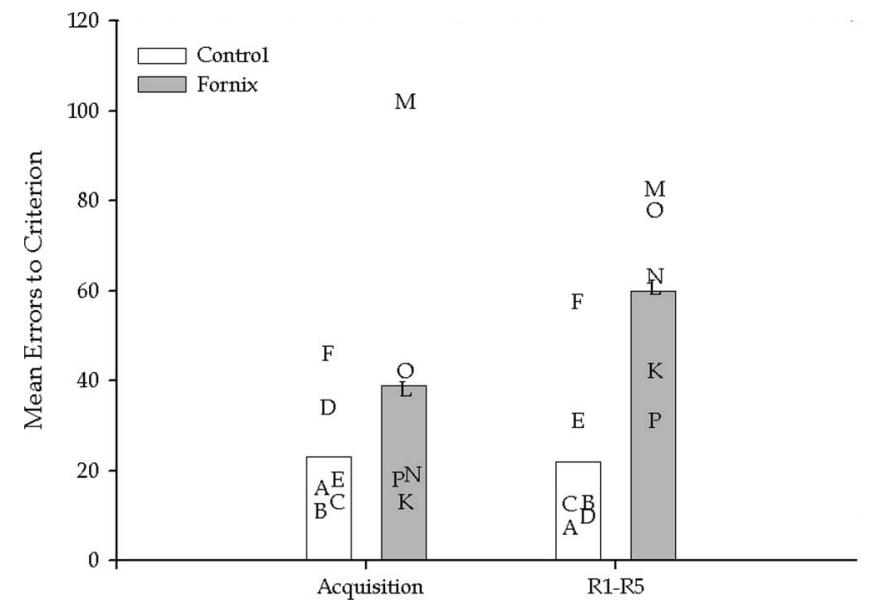

Figure 2. Mean errors to criterion made by monkeys in the acquisition stage and then the five subsequent reassignment stages plotted together (R1-R5). The letters represent scores of individual monkeys.

Table 3. Mean errors to criterion for the different shift types, as defined in the experimental procedure, over stages R1-R5

\begin{tabular}{lcccc}
\hline & Same & Reversal & Plus & Minus \\
\hline Group Fx & 38.3 & 112.7 & 118.8 & 27.2 \\
Group Con & 9.5 & 49.3 & 42.5 & 7.7 \\
\hline
\end{tabular}

the different types of shifts, perhaps as a consequence of scaling of the impairment on problems with different levels of difficulty, fornix transection produces a significant impairment on every type of shift.

\section{Discussion}

We have shown that transection of the fornix in the macaque monkey impairs a concurrent object discrimination task in which the reward assignments and pairings of stimuli in the task have been randomly reassigned relative to a previous stage of training. This impairment was significant in all types of reassignment, most notably including the Same shifts in which the stimuli were re-paired but critically retained the same response-reinforcement contingencies. This result is particularly notable considering that the initial learning of novel concurrent discriminations is not impaired. The latter finding replicates the results of Moss et al.

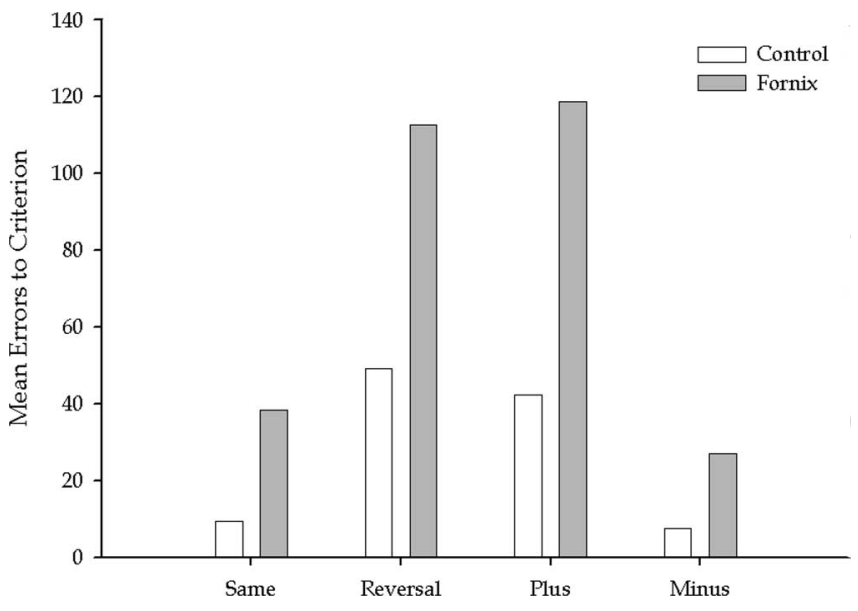

Figure 3. Mean errors to criterion made by monkeys in the five reassignment stages, separated by the shift types as defined in the methods section.

(1981), and also Gaffan (1994b), an experiment performed in the same apparatus as the current study. In the latter case, Gaffan showed in task 3 of his paper that concurrent object discrimination learning is not impaired after fornix transection in monkeys, in the context of constantly changing backgrounds and object locations that give no additional information about the nature of the rewarded object. This was therefore a robust test of pure object discrimination learning.

We chose to investigate the current task using fornix transection, rather than selective hippocampal lesions. Fornix transection interrupts the subcortical inputs and outputs of the hippocampus, and has previously been shown to have equivalent effects in a number of tasks to direct lesions to the hippocampus (Aggleton et al., 1992; Wible et al., 1992; Whishaw and Jarrard, 1995), although their effects are not always identical (McDonald et al., 1997). Importantly, transection of the fornix avoids damage to any of the cortical areas surrounding the hippocampus, particularly areas of rhinal cortex, and so may be a better way to produce selective hippocampal dysfunction than direct lesions.

A notable feature of the current task, compared with many others impaired by lesions to fornix and hippocampus, is that it contains no spatial demands whatsoever, as there were no spatial cues for learning, and stimulus positions were assigned random left-right positions on the screen throughout. This is not the first 
evidence for a nonspatial impairment after fornix transection in the monkey. Brasted et al. $(2002,2003)$ showed impairments in monkeys with fornix transection on conditional visuomotor learning in tasks involving nonspatially differentiated responses. Furthermore, Charles et al. (2004) showed that monkeys with fornix transection are impaired at making judgements of relative recency, but not of absolute novelty, an impairment in knowledge about temporal context of events. There is also similar evidence after hippocampal lesions in rats (Agster et al., 2002; Fortin et al., 2002).

Nevertheless, a wide range of literature in rats (O'Keefe and Nadel, 1978), monkeys (Gaffan, 1994a), and humans (Maguire et al., 1997) supports some form of spatial processing role for the hippocampus and fornix. Monkeys with fornix transection are impaired at spatial-visual conditional tasks (Gaffan and Harrison, 1989a, 1989b), spatial delayed nonmatching-to-sample (Murray et al., 1989), concurrent spatial discrimination (Buckley et al., 2004), and at learning "object-in-place" discriminations, in which a spatial context provided by a unique computer generated scene assists learning of an object discrimination (Gaffan, 1994b).

It seems, therefore, that adherence to a traditional view of the function of the fornix and hippocampus as spatial is an oversimplification, and a number of theories have attempted to characterize a generalized form for the representations used by the hippocampus to mediate such a wide variety of forms of learning, including those that generalize the function to the formation of configural (Rudy and Sutherland, 1995) or relational representations (Eichenbaum et al., 1994; Eichenbaum and Cohen, 2001). Previous work has proposed that relational memory encoding is dependent on the hippocampal system in monkeys, being specifically impaired by lesions that include entorhinal cortex (Buckmaster et al., 2004). The current study does not seek to discriminate between differing accounts of this nature, but it does contribute to the expanding body of work in support of a wide ranging role for the hippocampus.

An important feature of the current result is that the impairment after reassignment was significant regardless of the way in which the random reassignment occurred (i.e., regardless of switch type). Most notably, the trials in which response-reinforcement contingencies of individual items did not change, or Same shifts, also elicited a significant impairment. This particular element of the current impairment requires a specific explanation, one which does not need to make reference to changing reward contingencies.

In the experiment, when the monkey makes a choice, a number of other events occur at the same time, such as the presence of the other (not chosen) object, and the delivery or not of food reward. Together, these form a context in which that choice was made. Because the monkey is making a series of such choices throughout the experiment, knowledge of which context as described here is applicable to which choice can be thought of as knowledge of temporal context. For a monkey to successfully learn about a series of changes in the temporal context, and what contingencies are in effect at any particular point, he needs to have good memory for that temporal context. Without knowledge of the temporal context, he will have difficulty in applying the new relations between objects and rewards, and be unable to discern separate phases of the experiment. When this temporal context is changing but irrelevant to the task, as in task 3 in the study by Gaffan (1994b) in which the background and object locations are randomly varying, fornix transection has no effect on learning. But in the current task the temporal context is rele- vant, and a deficit in processing it may cause a disruption in the processing of events, regardless of the type of switch, and most notably even in the case where the reward contingencies of the two stimuli remain unchanged (Same shifts).

Hence, we propose that the impairment here may result from a general impairment in the processing of temporal context, because the only disruption in a same shift is the repairing of stimuli, which is a shift in the temporal context without a shift in reward assignments. The presence of the impairment in the same shifts is important, as it promotes the explanation in terms of temporal context, and argues against an ostensibly simpler one of remapping stimulus-reward contingencies, or learning the relative value of rewards. We appreciate, however, that the current task, in contrast to previous ones (Charles et al., 2004), did not contain an explicit requirement for remembering temporal context, and that alternative explanations can be provided for the impairments after the other types of shift.

If there is indeed an impairment in temporal context, it should not be regarded as an indicator of a generalized impairment in learning about context after fornix transection in the monkey. This is because a number of tasks, in which a context is formed by a nonspatial conditional cue to an object discrimination, are not impaired by fornix transection. Specifically, the use of information about the context created by a cue in the form of an auditory stimulus or food reward (Gaffan et al., 1984) or by a visual stimulus (Gaffan and Harrison, 1989a) is normal in monkeys with fornix transection.

Other alterations in stimulus processing, such as overunitization of discrimination problems (Bussey et al., 1998; Saksida et al., 2007) or an impairment in pattern separation of the stimulus pairings (Kesner and Hopkins, 2006), may be consistent with impaired learning of re-paired discrimination problems, especially those in which the reward associations of individual stimuli are the same as in the previous set. However, these views would seem to predict facilitation of repaired problems in which reward associations are inconsistent with the previous set, which is the opposite result to ours.

Regardless of whether our specific hypothesis is correct, the current study adds to the evidence that the fornix, and by extension the hippocampus, has a role that is not merely limited to the spatial domain.

\section{References}

Aggleton JP, Keith AB, Rawlins JN, Hunt PR, Sahgal A (1992) Removal of the hippocampus and transection of the fornix produce comparable deficits on delayed non-matching to position by rats. Behav Brain Res 52:61-71.

Agster KL, Fortin NJ, Eichenbaum H (2002) The hippocampus and disambiguation of overlapping sequences. J Neurosci 22:5760-5768.

Brasted PJ, Bussey TJ, Murray EA, Wise SP (2002) Fornix transection impairs conditional visuomotor learning in tasks involving nonspatially differentiated responses. J Neurophysiol 87:631-633.

Brasted PJ, Bussey TJ, Murray EA, Wise SP (2003) Role of the hippocampal system in associative learning beyond the spatial domain. Brain 126:1202-1223.

Buckley MJ, Charles DP, Browning PG, Gaffan D (2004) Learning and retrieval of concurrently presented spatial discrimination tasks: role of the fornix. Behav Neurosci 118:138-149.

Buckmaster CA, Eichenbaum H, Amaral DG, Suzuki WA, Rapp PR (2004) Entorhinal cortex lesions disrupt the relational organization of memory in monkeys. J Neurosci 24:9811-9825.

Bussey TJ, Clea Warburton E, Aggleton JP, Muir JL (1998) Fornix lesions can facilitate acquisition of the transverse patterning task: a challenge for "configural" theories of hippocampal function. J Neurosci 18:1622-1631. Charles DP, Gaffan D, Buckley MJ (2004) Impaired recency judgments and 
intact novelty judgments after fornix transection in monkeys. J Neurosci 24:2037-2044.

Deacon RM, Rawlins JN (1996) Effects of aspiration lesions of hippocampus or overlying neocortex on concurrent and configural object discriminations in rats. Behav Brain Res 77:165-174.

Eichenbaum H, Cohen NJ (2001) From conditioning to conscious recollection: memory systems of the brain. New York: Oxford UP.

Eichenbaum H, Fagan A, Cohen NJ (1986) Normal olfactory discrimination learning set and facilitation of reversal learning after medialtemporal damage in rats: implications for an account of preserved learning abilities in amnesia. J Neurosci 6:1876-1884.

Eichenbaum H, Mathews P, Cohen NJ (1989) Further studies of hippocampal representation during odor discrimination learning. Behav Neurosci 103:1207-1216.

Eichenbaum H, Otto T, Cohen NJ (1994) Two functional components of the hippocampal memory system. Behav Brain Sci 17:449-518.

Fortin NJ, Agster KL, Eichenbaum HB (2002) Critical role of the hippocampus in memory for sequences of events. Nat Neurosci 5:458-462.

Gaffan D (1994a) Dissociated effects of perirhinal cortex ablation, fornix transection and amygdalectomy: evidence for multiple memory systems in the primate temporal lobe. Exp Brain Res 99:411-422.

Gaffan D (1994b) Scene-specific memory for objects: a model of episodic memory impairment in monkeys with fornix transection. J Cogn Neurosci 6:305-320.

Gaffan D, Harrison S (1989a) Place memory and scene memory: effects of fornix transection in the monkey. Exp Brain Res 74:202-212.

Gaffan D, Harrison S (1989b) A comparison of the effects of fornix transection and sulcus principalis ablation upon spatial learning by monkeys. Behav Brain Res 31:207-220.

Gaffan D, Saunders RC, Gaffan EA, Harrison S, Shields C, Owen MJ (1984) Effects of fornix transection upon associative memory in monkeys: role of the hippocampus in learned action. Q J Exp Psychol B 36:173-221.
Kesner RP, Hopkins RO (2006) Mnemonic functions of the hippocampus: a comparison between animals and humans. Biol Psychol 73:3-18.

Maguire EA, Frackowiak RSJ, Frith CD (1997) Recalling routes around London: activation of the right hippocampus in taxi drivers. J Neurosci 17:7103-7110.

McDonald RJ, Murphy RA, Guarraci FA, Gortler JR, White NM, Baker AG (1997) Systematic comparison of the effects of hippocampal and fornixfimbria lesions on acquisition of three configural discriminations. Hippocampus 7:371-388.

Moss M, Mahut H, Zola-Morgan S (1981) Concurrent discrimination learning of monkeys after hippocampal, entorhinal, or fornix lesions. J Neurosci 1:227-240.

Murray EA, Davidson M, Gaffan D, Olton DS, Suomi S (1989) Effects of fornix transection and cingulate cortical ablation on spatial memory in rhesus monkeys. Exp Brain Res 74:173-186.

O'Keefe J, Nadel L (1978) The hippocampus as a cognitive map. Oxford: Clarendon.

Rudy JW, Sutherland RJ (1995) Configural association theory and the hippocampal formation: an appraisal and reconfiguration. Hippocampus 5:375-389.

Saksida LM, Bussey TJ, Buckmaster CA, MurrayEA (2007) Impairment and facilitation of transverse patterning after lesions of the perirhinal cortex and hippocampus, respectively. Cereb Cortex 17:108-115.

Teng E, Stefanacci L, Squire LR, Zola SM (2000) Contrasting effects on discrimination learning after hippocampal lesions and conjoint hippocampal-caudate lesions in monkeys. J Neurosci 20:3853-3863.

Whishaw IQ, Jarrard LE (1995) Similarities vs. differences in place learning and circadian activity in rats after fimbria-fornix section or ibotenate removal of hippocampal cells. Hippocampus 5:595-604.

Wible CG, Shiber JR, Olton DS (1992) Hippocampus, fimbria-fornix, amygdala, and memory: object discriminations in rats. Behav Neurosci 106:751-761. 